\section{S,S-Dimethyl Dithiocarbonate: A Convenient Reagent for the Synthesis of Symmetrical and Unsymmetrical Ureas}

\author{
Man-kit Leung,* J un-Liang Lai, King-Hang Lau, \\ Hsiao-hua Yu, and Hsiang-J u Hsiao \\ Department of Chemistry, National Taiwan University, \\ Taipei, Taiwan, Republic of China
}

Received December 28, 1995

The condensation of primary amines with phosgene or isocyanates is a classic method for the synthesis of organic ureas. ${ }^{1}$ However, due to their high toxicity and reactivity, phosgene and isocyanates are difficult to handle in the laboratory. Although several substitutes for phosgene such as triphosgene and carbonyldiimidazole have been devel oped during the last few decades, ${ }^{2,3}$ these reagents are themselves prepared from phosgene. Alternative methods involving drastic reaction conditions, such as direct reactions of amines with dialkyl carbonates at high temperature $\mathrm{e}^{4}$ and the reaction of amines with $\mathrm{N}, \mathrm{N}^{\prime}$-diphenylurea in the presence of $\mathrm{Et}_{3} \mathrm{~N}$ in refluxing $D M F, 5$ have been reported previously. Our efforts are therefore directed toward the development of mild reagents that can be used instead of phosgene or its derivatives in urea synthesis. Since S,S-dimethyl dithiocarbonate (DMDTC) is structurally similar to phosgene and can be prepared from methanol, carbon disulfide, and dimethyl sulfate by a two-step sequence, ${ }^{6}$ DMDTC was deemed an appropriate candidate for our investigation. Although dimethyl sulfate is a suspected human carcinogen, the substance is relatively nonvolatile (bp $188^{\circ} \mathrm{C}$ ) and can be handled safely with care in the laboratory.

To explore the feasibility of using DMDTC in urea synthesis, we first reacted DMDTC with 2 equiv of primary alkyl amines 1, 3, and $\mathbf{5}$ using methanol or ethanol as the solvent at $60^{\circ} \mathrm{C}$ and obtained, respectively, the desired symmetrical ureas $\mathbf{2 , 4}$, and $\mathbf{6}$ as the only products (Table 1 ). No incorporation of methanol or ethanol into the products occurred according to the ${ }^{1} \mathrm{H}$ NMR analyses. While the above procedure is quite successful for primary alkylamines, we discovered that DMDTC is relatively sensitive to steric environment and the nucleophilicity of the amino group. Thus, $\alpha$-substituted amines 7 and 9 react with DMDTC at much slower rates. Furthermore, reaction of tert-butylamine with DMDTC does not proceed under the same conditions. Although piperidine (11) is considered to be a good nucleophile, it only reacts with DMDTC at a moderate rate, affording thiocarbamate $\mathbf{1 2}$ as the product. Attempts to extend our procedure to less nucleophilic aromatic amines such as aniline were unsuccessful, resulting in recovery of the starting materials. From the

(1) (a) Hegarty, A. F. In Comprehensive Organic Chemistry; Barton, D., Ollis, W. D., Eds.; Pergamon Press: New York, 1979; Vol. 2, p 1067. (b) Sandler, S. R.; Karo, W. Organic Functional Group Preparations; Academic Press: New York, 1971; Vol. 2, p 135.

(2) Staab, H. A. Angew. Chem., Int. Ed. Engl. 1962, 1, 531.

(3) Eckert, H.; Forster, B. Angew. Chem., Int. Ed. Engl. 1987, 26, 894.

(4) Flyes, T. M.; J ames, T. D.; Pryhitka, A.; Zojsji, M. J . Org. Chem. 1993, 58, 7456.

(5) Ramadas, K.; Srinivasan N. Org. Prep. Proc. 1993, 25, 600.

(6) (a) Degani, I.; Fochi, R.; Regondi, V. Synthesis 1980, 375. (b) Degani, I.; Fochi, R.; Regondi, V. Synthesis 1981, 149. above observations, we concluded that DMDTC is a very selective reagent toward amines. By taking advantage of its selectivity, we successfully obtained the bisaniline 14 directly from DMDTC and 4-aminobenzylamine (13) without the need for protection and deprotection procedures. Furthermore, heating DMDTC with 2 equiv of 3-aminopropanol (15) in methanol afforded a mixture of bis(3-hydroxypropyl)urea $\mathbf{1 6}$ and the cyclic urethane $\mathbf{1 7}$ in a ratio of 7:3. More interesting is the fact that $\mathbf{1 6}$ is a highly crystalline compound which was readily purified by recrystallization from chloroform. Nevertheless, because of the proximity of the amino group and the hydroxy group, preparation of bis(2-hydroxyethyl)urea (19) in methanol was less successful, rendering oxazolidone (20) as the major product. Since the product ratio of the symmetrical urea to the cyclic carbamate is dependent on the reaction concentration of the corresponding amino alcohol, we carried out the synthesis at higher concentration, obtaining the desired symmetrical bis(hydroxyalkyl)ureas 16 and 19 in satisfactory yields.

Further experiments with DMDTC (Table 2 ) revealed that aliphatic amines bearing a hydroxy or an amino substituent at the $\beta$ or $\gamma$ position react in dilute solution to provide predominantly cyclic ureas or carbamates. In particular, reaction of ( \pm )-3-aminopropane1,2-diol (21) with DMDTC afforded exclusively 5(hydroxymethyl)oxazolidin-2-one (22), the kinetically favored isomer. On the other hand, 1,3-diamino-2propanol (23) reacted under the same conditions to give $\mathbf{2 4}$ as the major product. Also formed was 5-(aminomethyl)oxazolidin-2-one (25) as a minor component. We attribute the regioselectivity of the cyclization to the greater nucleophilicity of the amino group, which favors the six-membered ring closure over oxazolidone formation.

In an effort to ascertain the scope of DMDTC application to the synthesis of unsymmetrical ureas, we examined the possibility of preparing $\mathrm{N}$-alkyl-S-methyl thiocarbamate by mono aminolysis of DMDTC. First, benzylamine was allowed to react with excess DMDTC (1.6 molar equiv), affording N-benzyl-S-methyl thiocarbamate (36) and dibenzylurea (6) in a ratio of 1:30. This result implies that the formation of dibenzylurea (6) at the second stage of the reaction is faster than $\mathrm{N}$-benzylS-methyl thiocarbamate (36) formation from DMDTC. To

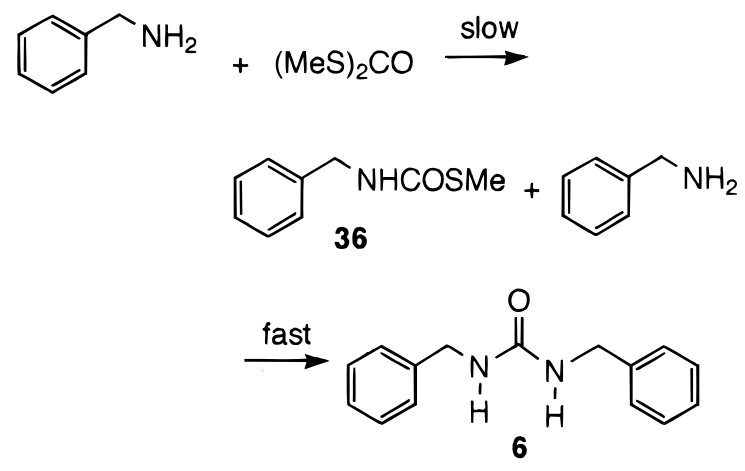

prevent thiocarbamate $\mathbf{3 6}$ from converting to dibenzylurea, we carried out the reaction under basic conditions, in the course of which $\mathbf{3 6}$ should be deprotonated immediately after being formed. Since the anion $\mathbf{3 7}$ is relatively stable toward nucleophilic substitution at ambient temperature and will not react further to give 
Table 1. Preparation of Symmetrical Ureas from Condensation of Various Amines with DMDTC

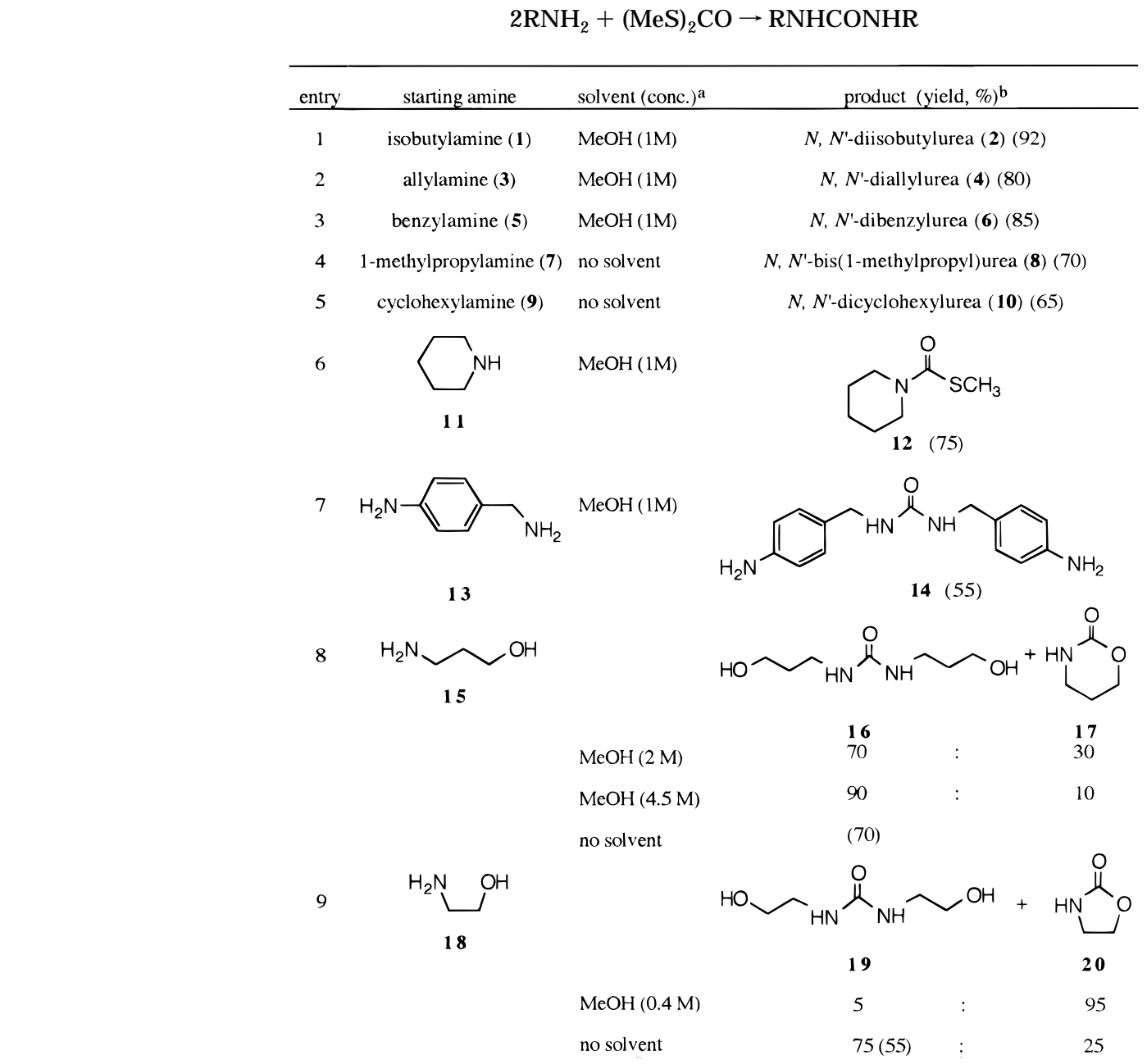

a I nitial concentration of the starting amine. ${ }^{b}$ I solated yield.

dibenzylurea (6), quenching of $\mathbf{3 7}$ led to the thiocarbamate $\mathbf{3 6}$ in high yield. Further condensation of $\mathbf{3 6}$ with

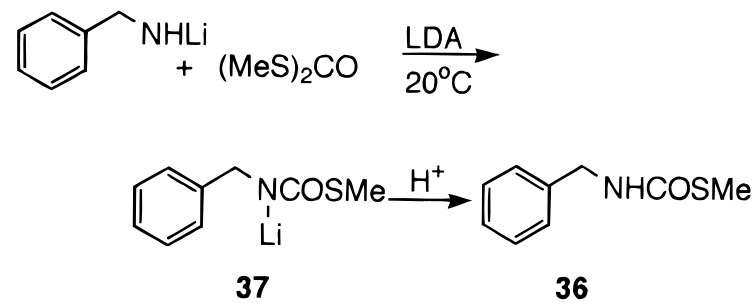

tetrahydrofurfurylamine furnished the unsymmetrical urea 38 (Table 3). This synthetic strategy was extended to the preparation of bisureas, a new class of guest-host molecules that has been devel oped recently for molecular recognition, ${ }^{7}$ by using bisthiocarbamate 39 as the key intermediate.

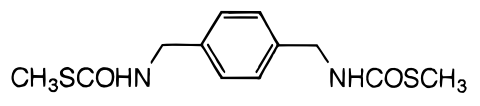

39

(7) (a) Nishizawa, S.; Bühlmann, P.; I wao, M.; Umezawa, Y Tetrahedron Lett. 1995, 36, 6483. (b) F'an, E.; Van Arman, S. A.; Kincaid, S.; Hamilton, A. D. J. Am. Chem. Soc. 1993, 115, 369. (c) Albert, J . S.; Hamilton, A. D. Tetrahedron Lett. 1993, 34, 7363.
The foregoing methodology offers a new approach to the synthesis of symmetrical and unsymmetrical ureas. It is particularly attractive for the preparation of hydroxy- and amino-substituted ureas that are not easy to obtain by conventional methods. In addition, unsymmetrical ureas have been prepared from DMDTC and two different amines through a two-step sequence. This method is especially useful when the corresponding isocyanates are unavailable.

\section{Experimental Section}

Materials. Amines (Aldrich, J anssan, Tokyo Kasei) were commercially available and used as received. S,S-Dimethyl dithiocarbonate (DMDTC) was prepared according to the procedures reported in the following section. Carbon disulfide is highly toxic, while benzene and dimethyl sulfate are suspected human carcinogens; these reagents should be handled in a properly ventilated fume hood, and rubber gloves should be worn.

Preparation of S,S-Dimethyl Dithiocarbonate (DMDTC). To a suspension of granulated $\mathrm{KOH}(21.5 \mathrm{~g}, 0.33 \mathrm{~mol})$ in anhydrous $\mathrm{Et}_{2} \mathrm{O}$ and benzene $(1: 1,140 \mathrm{~mL})$ was added methanol $(10.5 \mathrm{~g}, 0.33 \mathrm{mmol})$, followed by dropwise addition of a solution of $\mathrm{CS}_{2}(24.7 \mathrm{~g}, 0.33 \mathrm{~mol})$ in benzene $(15 \mathrm{~mL})$ at $0{ }^{\circ} \mathrm{C}$. The reaction mixture was kept at $0{ }^{\circ} \mathrm{C}$ for $5 \mathrm{~h}$, and $\mathrm{Me}_{2} \mathrm{SO}_{4}(41$ $\mathrm{g}, 0.33 \mathrm{~mol}$ ) was added. The reaction was allowed to react at ambient temperature for $20 \mathrm{~h}$. The organic layer then was decanted, washed sequentially with dilute $\mathrm{HCl}$ solution and saturated $\mathrm{NaCl}$ solution, dried over anhydrous $\mathrm{Na}_{2} \mathrm{SO}_{4}$, and 
Table 2. Condensation of Various Diamines or Amino Alcohols with DMDTC

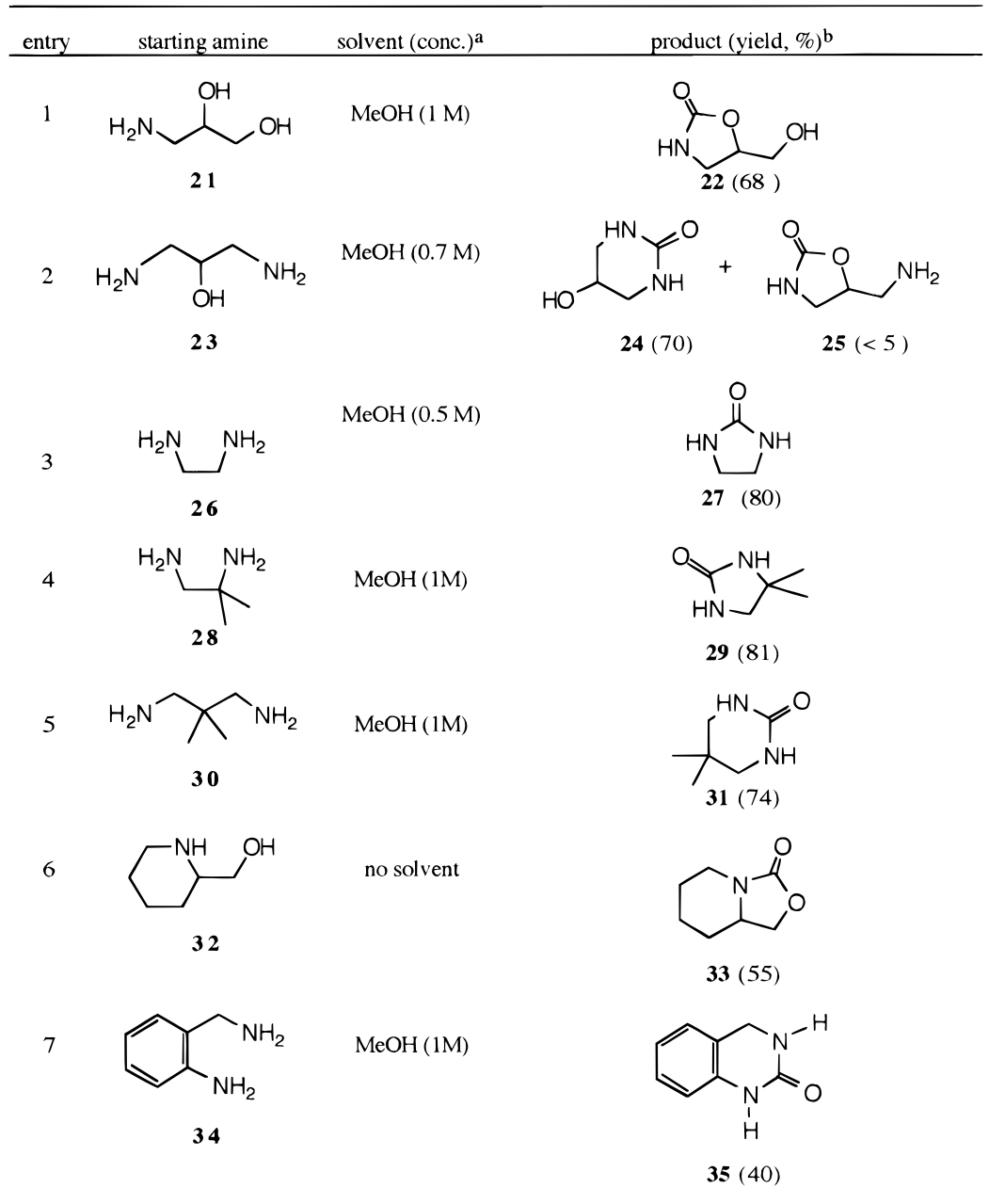

a I nitial concentration of the starting amine. ${ }^{b}$ I solated yield.

concentrated using simple distillation to afford crude O,Sdimethyl dithiocarbonate $(39 \mathrm{~g})$. The oily crude product was then subjected to thermal rearrangement at $100-110^{\circ} \mathrm{C}$, using tetrabutylammonium iodide ( $3 \mathrm{~mol} \%$ ) as a catalyst. After being heated for $18 \mathrm{~h}$, the yellowish crude oil was fractionally distilled under reduced pressure to give DMDTC as a colorless oil ( $25 \mathrm{~g}$, 58\%): bp $60-62{ }^{\circ} \mathrm{C}(20 \mathrm{mmHg})$ (lit. ${ }^{6}$ bp $\left.58-59^{\circ} \mathrm{C}, 16 \mathrm{Torr}\right){ }^{1}{ }^{1} \mathrm{H}$ NMR $\left(200 \mathrm{MHz}, \mathrm{CDCl}_{3}\right) \delta 2.43(\mathrm{~s}, 6 \mathrm{H})\left(\mathrm{lit}^{6}{ }^{1} \mathrm{H} \mathrm{NMR}\left(\mathrm{CCl}_{4}\right) \delta\right.$ $2.40(\mathrm{~s}, 6 \mathrm{H})) ;{ }^{13} \mathrm{C} \mathrm{NMR}\left(75 \mathrm{MHz} \mathrm{CDCl}_{3}\right) \delta 189.8,12.7$.

Typical Synthetic Procedures for Symmetrical Ureas. N,N'-Bis(isobutyl)urea (2) (Method A). To a stirred solution of isobutylamine (1) $(0.18 \mathrm{~g}, 2.6 \mathrm{mmol})$ in methanol $(1.5 \mathrm{~mL})$ was added DMDTC $(0.16 \mathrm{~g}, 1.3 \mathrm{mmol})$. The mixture was heated at $60{ }^{\circ} \mathrm{C}$ for $24 \mathrm{~h}$. The released malodorous methyl sulfide by product was absorbed and oxidized by $\mathrm{NaOCl}$ solution. When the reaction was complete, the reaction mixture was concentrated under reduced pressure, providing a crude solid which was further purified by recrystallization from methanol $-\mathrm{H}_{2} \mathrm{O}$ (1:1) to give 2 as colorless crystals (92\%): $\mathrm{mp} 130-132{ }^{\circ} \mathrm{C}$ (lit. ${ }^{8}$ 128-130 $\left.{ }^{\circ} \mathrm{C}\right) ;{ }^{1} \mathrm{H}$ NMR $\left(200 \mathrm{MHz} \mathrm{CDCl}_{3}\right) \delta 5.54$ (broad triplet, $2 \mathrm{H}), 2.91(\mathrm{t}, \mathrm{J}=6.1 \mathrm{~Hz}, 4 \mathrm{H}), 1.72-1.56(\mathrm{~m}, 2 \mathrm{H}), 0.84(\mathrm{~d}, \mathrm{~J}=$ $6.6 \mathrm{~Hz}, 12 \mathrm{H}) ;{ }^{13} \mathrm{C} \mathrm{NMR}\left(75 \mathrm{MHz} \mathrm{CDCl}_{3}\right) \delta 159.4,47.7,29.0$, 20.1; IR (KBr) cm $\mathrm{cm}^{-1} 3347,1623,1572 ; \mathrm{MS}$ (EI, $\left.70 \mathrm{eV}\right) \mathrm{m} / \mathrm{e} 172$ $\left(\mathrm{M}^{+}, 100\right), 157\left(\mathrm{M}^{+}-\mathrm{CH}_{3}, 20\right), 129\left(\mathrm{M}^{+}-\mathrm{C}_{3} \mathrm{H}_{7}, 25\right), 72(29), 58$ (81); HRMS calcd for $\mathrm{C}_{9} \mathrm{H}_{20} \mathrm{~N}_{2} \mathrm{O}$ 172.1576, obsd 172.1573. Anal. Calcd for $\mathrm{C}_{9} \mathrm{H}_{20} \mathrm{~N}_{2} \mathrm{O}: \mathrm{C}, 62.75 ; \mathrm{H}, 11.70 ; \mathrm{N}$ : 16.26. Found: C, 62.49; H, 11.67; N; 16.21.

$\mathbf{N}, \mathbf{N}^{\prime}$-Diallylurea (4): colorless crystals; mp 93-95 ${ }^{\circ} \mathrm{C}$ (lit. ${ }^{9}$ $\left.\mathrm{mp} 92-94^{\circ} \mathrm{C}\right)$.

(8) Franz, R. A.; Applegath, F.; Morriss, F. V.; Baiocchi, F. J . Org. Chem. 1961, 26, 3306.

(9) Laufer, D. A.; Al-Farhan, E. J . Org. Chem. 1991, 56, 891.
$\mathbf{N}, \mathbf{N}^{\prime}$-Dibenzylurea (6). Recrystallization from $\mathrm{CHCl}_{3}-$ hexane yielded $\mathbf{6}$ as colorless crystals: $\mathrm{mp} 166-168{ }^{\circ} \mathrm{C}$ (lit. ${ }^{10}$ $\left.\mathrm{mp} 167-170{ }^{\circ} \mathrm{C}\right)$.

$\mathbf{N}, \mathbf{N}$-Cyclohexylurea (10). Recrystallization from $\mathrm{MeOH}$ yiel ded $\mathbf{1 0}$ as colorless crystals: $\mathrm{mp} 230-231^{\circ} \mathrm{C}$ (lit. ${ }^{\mathrm{lb}} \mathrm{mp} 229$ $230{ }^{\circ} \mathrm{C}$ from $\mathrm{MeOH}$ ).

S-Methyl 1-Piperidinecarbothioate (12). Flash chromatography on silica gel, using $\mathrm{MeOH}-\mathrm{CH}_{2} \mathrm{Cl}_{2}$ (1:7) as the eluent, afforded 12 as a colorless oil: ${ }^{1} \mathrm{H}$ NMR $\left(200 \mathrm{M} \mathrm{Hz} \mathrm{CDCl}_{3}\right) \delta 3.52-$ $3.40(\mathrm{~m}, 4 \mathrm{H}), 2.32(\mathrm{~s}, 3 \mathrm{H}), 1.70-1.49(\mathrm{~m}, 6 \mathrm{H}) ;{ }^{13} \mathrm{C} \mathrm{NMR}(50 \mathrm{MHz}$, $\left.\mathrm{CDCl}_{3}\right) \delta 167.1,45.7$ (broad peak), 25.6, 24.4, 12.8; IR (neat, $\mathrm{NaCl}) \mathrm{cm}^{-1}$ 1734; $\mathrm{MS}$ (EI, $\left.70 \mathrm{eV}\right) \mathrm{m} / \mathrm{e} 159\left(\mathrm{M}^{+}, 28\right), 112\left(\mathrm{M}^{+}-\right.$ $\mathrm{CH}_{3} \mathrm{~S}, 81$ ), 69 (100); HRMS (EI, $70 \mathrm{eV}$ ) calcd for $\mathrm{C}_{7} \mathrm{H}_{13} \mathrm{NOS}$ 159.0719, obsd 159.0722.

N,N'-Bis(4-aminobenzyl)urea (14). Flash chromatography on silica gel, using $\mathrm{MeOH}-\mathrm{CHCl}_{3}(1: 20)$ as the eluent afforded 14 as colorless crystals: mp $200-202{ }^{\circ} \mathrm{C} ;{ }^{1} \mathrm{H}$ NMR $(200 \mathrm{MHz}$, DMSO-d 6 ) $\delta 6.90(\mathrm{~d}, \mathrm{~J}=8.2 \mathrm{~Hz}, 4 \mathrm{H}), 6.50(\mathrm{~d}, \mathrm{~J}=8.2 \mathrm{~Hz}, 4 \mathrm{H})$, 6.04 (broad triplet, $5.6 \mathrm{~Hz}, 2 \mathrm{H}$ ), 4.90 (bs, 4H), 4.02 (d, J = 5.6 $\mathrm{Hz}, 4 \mathrm{H}) ;{ }^{13} \mathrm{C}$ NMR (50 MHz, DMSO-d 6 ) $\delta 158.2,147.4,128.2$, 127.8, 113.9, 43.0; IR (KBr) cm-1 3416, 3318, 1606, 1560; MS (El, $70 \mathrm{eV}) \mathrm{m} / \mathrm{e} 270\left(\mathrm{M}^{+}, 40\right), 177$ (32), 164 (61), 121 (100), 106 (45), 94 (20); HRMS (EI, $70 \mathrm{eV)}$ calcd for $\mathrm{C}_{15} \mathrm{H}_{18} \mathrm{~N}_{4} \mathrm{O} 270.1480$, obsd 270.1479. Anal. Calcd for $\mathrm{C}_{15} \mathrm{H}_{18} \mathrm{~N}_{4} \mathrm{O}$ : C, 66.64; $\mathrm{H}, 6.71$; N, 20.72. Found: C, 66.34; $\mathrm{H}, 6.67 ; \mathrm{N}, 20.59$.

N,N'-Bis(2-hydroxyethyl)urea (19) (Method B). Excess ethanolamine and DMDTC were mixed and heated at $60{ }^{\circ} \mathrm{C}$ for $15 \mathrm{~h}$. The released malodorous methyl sulfide by product was absorbed and oxidized by $\mathrm{NaOCl}$ solution. When the reaction was complete, the unreacted ethanolamine was removed under reduced pressure by simple distillation, providing a crude

(10) Pihuleac, J .; Bauer, L. Synthesis 1989, 61. 
Table 3. Preparation of Unsymmetrical Ureas from DMDTC

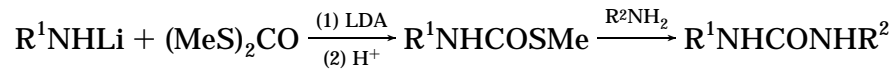

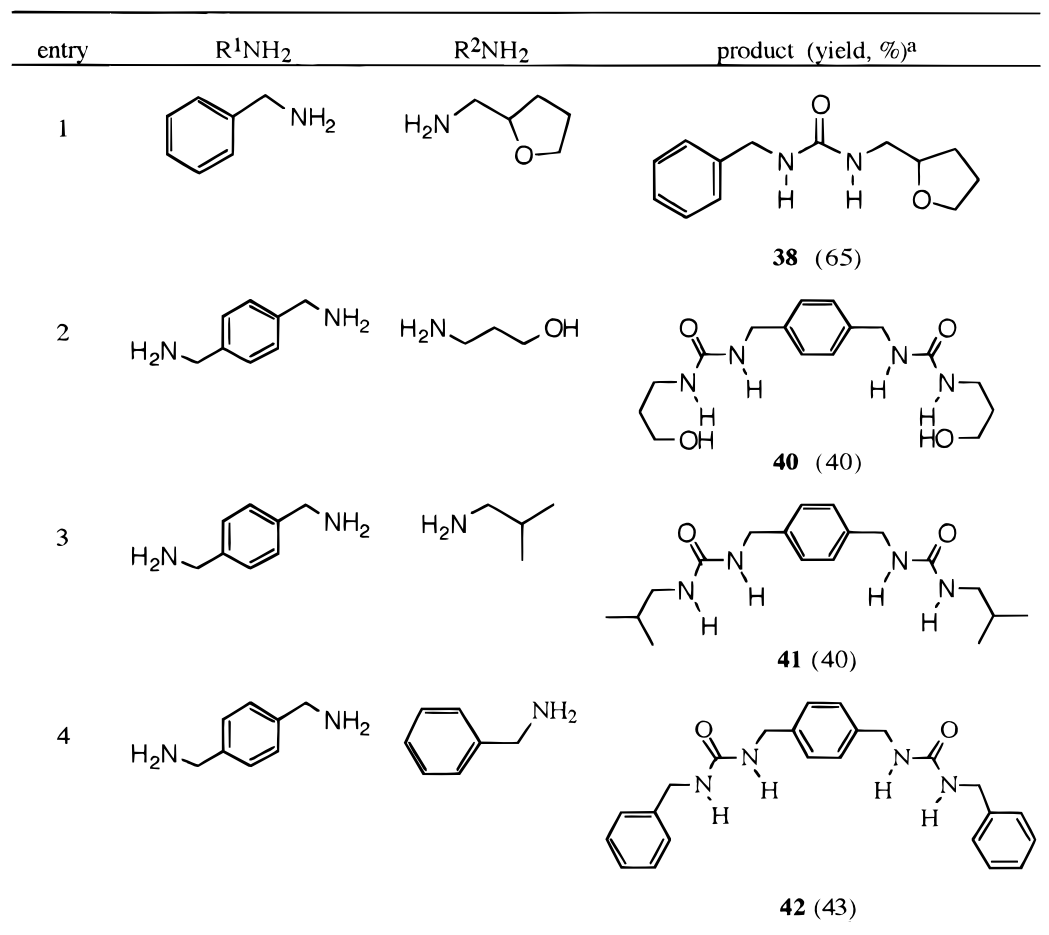

a Overall isolated yield.

mixture of oxazolidone and bis(2-hydroxyethyl)urea which was further purified by recrystallization from methanol-ethyl acetate (1:4.5) to give 19 as colorless crystals: $\mathrm{mp} 82-84{ }^{\circ} \mathrm{C} ;{ }^{1} \mathrm{H}$ NMR (200 MHz, DMSO-d 6 ) $\delta 5.96$ (broad triplet, 2H), 4.62 (bs, $2 \mathrm{H}), 3.33$ (bs, 4H), $3.02(\mathrm{q}, \mathrm{J}=6 \mathrm{~Hz}, 4 \mathrm{H}) ;{ }^{13} \mathrm{C} \mathrm{NMR}(75 \mathrm{MHz}$, DMSO-d 6 ) $\delta$ 158.4, 60.8, 42.1; IR $(\mathrm{KBr}) \mathrm{cm}^{-1}$ 3343, 1621; MS $(E I, 70 \mathrm{eV}) \mathrm{m} / \mathrm{e} 149\left(\mathrm{M}^{+}+\mathrm{H}, 5\right), 148\left(\mathrm{M}^{+}, 2\right), 118$ (100), 117 (60), 70 (40); HRMS (EI, $70 \mathrm{eV}$ ) calcd for $\mathrm{C}_{5} \mathrm{H}_{12} \mathrm{~N}_{2} \mathrm{O}_{3} 148.0848$, obsd 148.0842. Anal. Calcd for $\mathrm{C}_{5} \mathrm{H}_{12} \mathrm{~N}_{2} \mathrm{O}_{3}$ : C, 40.53; $\mathrm{H}, 8.17$; N, 18.91. Found: C, 40.08; H, 8.16; N, 18.78.

N,N'-Bis(3-hydroxypropyl)urea (16). Recrystallization from $\mathrm{MeOH}-\mathrm{CHCl}_{3}$ provided $\mathbf{1 6}$ as colorless crystals: $\mathrm{mp} 83-86^{\circ} \mathrm{C}$ (lit. ${ }^{4} \mathrm{mp} 93-94{ }^{\circ} \mathrm{C}$ from $\mathrm{CH}_{3} \mathrm{CN}$ ); ${ }^{1} \mathrm{H}$ NMR (200 MHz, DMSO$\left.\mathrm{d}_{6}\right) \delta 5.82(\mathrm{t}, \mathrm{J}=6.0 \mathrm{~Hz}, 2 \mathrm{H}), 4.00-4.50(\mathrm{bs}, 2 \mathrm{H}), 3.38(\mathrm{t}, \mathrm{J}=$ $6.0 \mathrm{~Hz}, 4 \mathrm{H}$ ), $3.00(\mathrm{q}, \mathrm{J}=6 \mathrm{~Hz}, 4 \mathrm{H}), 1.48$ (quintet, J $=6 \mathrm{~Hz}, 4 \mathrm{H}$ ) (lit. ${ }^{1}{ }^{\mathrm{H}} \mathrm{NMR}\left(90 \mathrm{MHz}, \mathrm{D}_{2} \mathrm{O}\right) \delta 3.5(\mathrm{t}, \mathrm{J}=7.0 \mathrm{~Hz}, 4 \mathrm{H}), 3.1(\mathrm{q}$, J $=7 \mathrm{~Hz}, 4 \mathrm{H}), 1.6(\mathrm{~m}, 4 \mathrm{H})) ;{ }^{13} \mathrm{C}$ NMR $\left(50 \mathrm{MHz}, \mathrm{DMSO}-\mathrm{d}_{6}\right) \delta 158.5$, 58.4, 36.4, 33.2 (lit. ${ }^{4}{ }^{13} \mathrm{C}$ NMR $\left(62.89 \mathrm{MHz}, \mathrm{D}_{2} \mathrm{O}\right) \delta 160.7,59.2$, 36.7, 31.8); IR (KBr) cm $\mathrm{cm}^{-1} 3339$ (br), 1613, 1584; MS (EI, $\left.70 \mathrm{eV}\right)$ $\mathrm{m} / \mathrm{e} 176\left(\mathrm{M}^{+}, 20\right), 146(65), 132(90), 102(40), 74(100), 57(91)$; HRMS (EI, $70 \mathrm{eV}$ ) calcd for $\mathrm{C}_{7} \mathrm{H}_{16} \mathrm{~N}_{2} \mathrm{O}_{3}$ 176.1161, obsd 176.1155 .

$\mathbf{N}, \mathbf{N}^{\prime}$-Bis(1-methylpropyl)urea (8). Recrystallization from $\mathrm{CHCl}_{3}$-hexane yielded 8 as colorless crystals: $\mathrm{mp}$ 131-132.5 ${ }^{\circ} \mathrm{C}$ (lit. $\left.{ }^{8} \mathrm{mp} 135^{\circ} \mathrm{C}\right) ;{ }^{1} \mathrm{H}$ NMR $\left(300 \mathrm{MHz}, \mathrm{CDCl}_{3}\right) \delta 4.30$ (bs, $\left.2 \mathrm{H}\right)$, $3.62(\mathrm{~m}, 2 \mathrm{H}), 1.48(\mathrm{~m}, 4 \mathrm{H}), 1.07(\mathrm{~d}, \mathrm{~J}=6.7 \mathrm{~Hz}, 6 \mathrm{H}), 0.87(\mathrm{t}, \mathrm{J}=$ $7.4 \mathrm{~Hz}, 6 \mathrm{H}) ;{ }^{13} \mathrm{C}$ NMR $\left(75 \mathrm{MHz} \mathrm{CDCl}_{3}\right) \delta 157.6,47.3,30.3,21.0$, 10.3; IR (KBr) cm 3339 (br), 1613, 1584; MS (EI, $70 \mathrm{eV}) \mathrm{m} / \mathrm{e}$ $172\left(\mathrm{M}^{+}, 12\right), 143\left(\mathrm{M}^{+}-\mathrm{C}_{2} \mathrm{H}_{5}, 63\right), 72(20), 58(100)$; HRMS (EI, $70 \mathrm{eV}$ ) calcd for $\mathrm{C}_{9} \mathrm{H}_{20} \mathrm{~N}_{2} \mathrm{O}$ 172.1576, obsd 172.1573. Anal. Calcd for $\mathrm{C}_{9} \mathrm{H}_{20} \mathrm{~N}_{2} \mathrm{O}$ : C, 62.74; $\mathrm{H}, 11.70 ; \mathrm{N}, 16.26$. Found: $\mathrm{C}, 62.49$; $\mathrm{H}, 11.57$; N, 16.39 .

Typical Synthetic Procedures for Cyclic Ureas and Carbamates. 5-(Hydroxymethyl)oxazolidin-2-one (22). To a stirred solution of 3-aminopropane-1,2-diol (21) in methanol was added DMDTC. After being heated at $60^{\circ} \mathrm{C}$ for $24 \mathrm{~h}$, the reaction mixture was concentrated under reduced pressure, providing a crude oil which was further purified by flash chromatography, using $\mathrm{MeOH}-\mathrm{CH}_{2} \mathrm{Cl}_{2}(1: 10)$ as the eluent to give 22 as colorless oil: ${ }^{1} \mathrm{H}$ NMR $\left(200 \mathrm{MHz}\right.$, DMSO- $\left.\mathrm{d}_{6}\right) \delta 7.38$ (bs, $1 \mathrm{H}), 5.06(\mathrm{t}, \mathrm{J}=5.6 \mathrm{~Hz}, 1 \mathrm{H}), 4.45-4.57(\mathrm{~m}, 1 \mathrm{H}), 3.33-3.58$ $(\mathrm{m}, 3 \mathrm{H}), 3.21(\mathrm{dd}, \mathrm{J}=8.4,6.6 \mathrm{~Hz}, 1 \mathrm{H})\left(\mathrm{lit} .{ }^{1}{ }^{1} \mathrm{H}\right.$ NMR $\left(\mathrm{CD}_{3} \mathrm{OD}\right) \delta$ $4.8(\mathrm{bs}, 2 \mathrm{H}), 4.6(\mathrm{~m}, 1 \mathrm{H}), 3.25-3.80(\mathrm{~m}, 4 \mathrm{H})) ;{ }^{13} \mathrm{C} \mathrm{NMR}(50 \mathrm{MHz}$, DMSO-d 6 ) $\delta 159.4,76.4,62.3,41.6$ (lit. ${ }^{11}{ }^{13} \mathrm{C} \mathrm{NMR}\left(\mathrm{CD}_{3} \mathrm{OD}\right) \delta$ 78.5, 63.5, 42.9); IR (KBr) cm ${ }^{-1} 3320(\mathrm{br}), 3297,1731$; MS (EI, $20 \mathrm{eV}) \mathrm{m} / \mathrm{e} 118\left(\mathrm{M}^{+}+\mathrm{H}, 5\right), 100\left(\mathrm{M}^{+}-\mathrm{OH}, 24\right), 86\left(\mathrm{M}^{+}-\mathrm{CH}_{2^{-}}\right.$ $\mathrm{OH}, 100) ; \mathrm{HRMS}(\mathrm{EI}, 70 \mathrm{eV})$ calcd for $\mathrm{C}_{4} \mathrm{H}_{7} \mathrm{NO}_{3} 117.0426$, obsd 117.0420.

Tetrahydro-5-hydroxy-2(1H)-pyrimidinone (24). Recrystallization of the crude product from $\mathrm{MeOH}$-acetone afforded 24 as colorless crystals: mp $210-213{ }^{\circ} \mathrm{C} ;{ }^{1} \mathrm{H}$ NMR $(200 \mathrm{MHz}$, DMSO-d 6 ) $\delta 6.16(\mathrm{bs}, 2 \mathrm{H}), 5.07(\mathrm{~d}, \mathrm{~J}=4 \mathrm{~Hz}, 1 \mathrm{H}), 3.79-3.82(\mathrm{~m}$ $1 \mathrm{H}), 3.05-3.20(\mathrm{~m}, 2 \mathrm{H}), 2.80-3.00(\mathrm{~m}, 2 \mathrm{H}) ;{ }^{13} \mathrm{C}$ NMR $(50 \mathrm{MHz}$, DMSO- $\left.\mathrm{d}_{6}\right) \delta 156.1,60.4,46.2 ; \mathrm{IR}(\mathrm{KBr}) \mathrm{cm}^{-1} 3344,3297,3217$ 1645; MS (EI, 20 eV) m/e 116 (M+, 90), 88 (30), 59 (100); HRMS (EI, $70 \mathrm{eV}$ ) calcd for $\mathrm{C}_{4} \mathrm{H}_{8} \mathrm{~N}_{2} \mathrm{O}_{2}$ 116.0586, obsd 116.0583. Anal. Calcd for $\mathrm{C}_{4} \mathrm{H}_{8} \mathrm{~N}_{2} \mathrm{O}_{2}:$ C, 41.37; $\mathrm{H}, 6.94 ; \mathrm{N}, 24.13$. Found: $\mathrm{C}$, 41.03; H, 6.84; N, 23.99.

4,4-Dimethyl-2-imidazolidinone (29). Recrystallization from $\mathrm{CH}_{2} \mathrm{Cl}_{2}$-hexane (4:1) afforded 29 as col orless crystals: $\mathrm{mp}$ 139-141 ${ }^{\circ} \mathrm{C}$; ${ }^{1} \mathrm{H}$ NMR (200 MHz, $\left.\mathrm{CDCl}_{3}\right) \delta 5.63$ (bs, 2H), 3.20

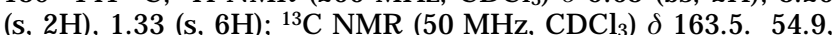
53.8, 28.1; IR ( $\mathrm{KBr}) \mathrm{cm}^{-1}$ 3281, 1704, 1688; MS (EI, $\left.70 \mathrm{eV}\right) \mathrm{m} / \mathrm{e}$ $114\left(\mathrm{M}^{+}, 10\right), 99$ (100), 56 (30); HRMS (EI, $\left.70 \mathrm{eV}\right)$ calcd for $\mathrm{C}_{5} \mathrm{H}_{10} \mathrm{~N}_{2} \mathrm{O}$ 114.0793, obsd 114.0787. Anal. Calcd for $\mathrm{C}_{5} \mathrm{H}_{10} \mathrm{~N}_{2} \mathrm{O}$ : C, 52.61; $\mathrm{H}, 8.83 ; \mathrm{N}, 24.54$. Found: $\mathrm{C}, 52.28 ; \mathrm{H}, 8.81$; $\mathrm{N}: 24.42$.

Tetrahydro-5,5-dimethyl-2(1H)-pyrimidinone (31). Recrystallization of $\mathbf{3 1}$ from $\mathrm{CH}_{2} \mathrm{Cl}_{2}$-hexane afforded colorless crystals: $\mathrm{mp} 254-256^{\circ} \mathrm{C}$ (lit. $\left.{ }^{12} 255-257^{\circ} \mathrm{C}\right) ;{ }^{1} \mathrm{H}$ NMR $(200 \mathrm{MHz}$, $\left.\mathrm{CDCl}_{3}\right) \delta 5.19(\mathrm{bs}, 2 \mathrm{H}), 2.94(\mathrm{~d}, \mathrm{~J}=2.2 \mathrm{~Hz}, 4 \mathrm{H}), 1.05(\mathrm{~s}, 6 \mathrm{H})$; ${ }^{13} \mathrm{C} \mathrm{NMR}\left(50 \mathrm{MHz} \mathrm{CDCl}_{3}\right) \delta 157.0,52.3,28.3,24.7$; IR $(\mathrm{KBr})$ $\mathrm{cm}^{-1}$ 3228, 1697; MS (EI, $\left.70 \mathrm{eV}\right) \mathrm{m} / \mathrm{e} 128\left(\mathrm{M}^{+}, 70\right), 113\left(\mathrm{M}^{+}-\right.$ $\mathrm{CH}_{3}$ ), 56 (100); $\mathrm{HRMS}(\mathrm{EI}, 70 \mathrm{eV})$ calcd for $\mathrm{C}_{6} \mathrm{H}_{12} \mathrm{~N}_{2} \mathrm{O} 128.0950$, obsd 128.0950. Anal. Calcd for $\mathrm{C}_{6} \mathrm{H}_{12} \mathrm{~N}_{2} \mathrm{O}$ : C, 56.23; $\mathrm{H}, 9.44$; N, 21.86. Found: C, 55.96; $\mathrm{H}, 9.40 ; \mathrm{N}, 21.76$.

Hexahydro-3H-oxazolo[3,4-a]pyridin-3-one (33). Flash chromatography on silica gel, using $\mathrm{MeOH}-\mathrm{CH}_{2} \mathrm{Cl}_{2}$ as the

(11) Cardillo, G.; Orena, M.; Sandri, S.; Tomasini, C. Tetrahedron 1987, 43, 2505

(12) Skinner, G. S., Hall, R. H.; Susi, P. V. J . Am. Chem. Soc. 1957 79, 3786. 
eluent, yielded 33 as a colorless oil: ${ }^{1} \mathrm{H} N M R\left(200 \mathrm{MHz}^{\mathrm{C}} \mathrm{CDCl}_{3}\right)$ $\delta 4.38(\mathrm{t}, 8.1 \mathrm{~Hz}, 1 \mathrm{H}), 3.88-3.96(\mathrm{~m}, 2 \mathrm{H}), 3.50-3.70(\mathrm{~m}, 1 \mathrm{H})$, $2.90-2.70(\mathrm{~m}, 1 \mathrm{H}), 1.78-1.98(\mathrm{~m}, 2 \mathrm{H}), 1.66-1.75(\mathrm{~m}, 1 \mathrm{H}), 1.20-$ $1.55(\mathrm{~m}, 3 \mathrm{H})$ (lit. ${ }^{13}{ }^{1} \mathrm{H}$ NMR (neat) $\delta 4.8(\mathrm{~m}, 1 \mathrm{H}), 4.2(\mathrm{~m}, 3 \mathrm{H})$, $3.25(\mathrm{~m}, 1 \mathrm{H}), 2.1(\mathrm{~m}, 6 \mathrm{H})) ;{ }^{13} \mathrm{C}$ NMR $\left(50 \mathrm{MHz}, \mathrm{CDCl}_{3}\right) \delta 156.8$, 67.9, 54.2, 41.1, 30.2, 24.0, 22.3; IR (neat, $\mathrm{NaCl}$ ) $\mathrm{cm}^{-1} 1739$; $\mathrm{MS}$ (EI, $70 \mathrm{eV}) \mathrm{m} / \mathrm{e} 141\left(\mathrm{M}^{+}, 100\right), 126(20), 97(30), 83$ (95), 69 (40), 55 (55); HRMS (EI, $70 \mathrm{eV}$ ) calcd to $\mathrm{C}_{7} \mathrm{H}_{11} \mathrm{NO}_{2}$ 141.0790, found 141.0799.

3,4-Dihydro-2(1H)-quinazolinone (35). Recrystallization of 35 from $\mathrm{MeOH}-\mathrm{CH}_{2} \mathrm{Cl}_{2}$ led to colorless crystals: $\mathrm{mp} \mathrm{222-}$ $223{ }^{\circ} \mathrm{C} ;{ }^{1} \mathrm{H}$ NMR $\left(200 \mathrm{MHz}, \mathrm{DMSO}^{\left.-\mathrm{d}_{6}\right)} \delta 8.98\right.$ (bs, $\left.1 \mathrm{H}\right), 7.03-$ $7.14(\mathrm{~m}, 2 \mathrm{H}), 6.78-6.87(\mathrm{~m}, 3 \mathrm{H}), 4.28(\mathrm{~s}, 2 \mathrm{H})$ (lit. ${ }^{14}{ }^{1} \mathrm{H}$ NMR (DMSO-d 6 ) $\delta 9.08$ (bs, 1H), 6.80-7.50 (m, 5H), $4.40(\mathrm{~s}, 2 \mathrm{H})) ;{ }^{13} \mathrm{C}$ NMR $\left(50 \mathrm{MHz}, \mathrm{DMSO}-\mathrm{d}_{6}\right) \delta 154.8,138.3,127.8,125.9,121.1$, 118.3, 113.7, 42.7; IR (KBr) cm $\mathrm{cm}^{-1}$ 3248, 1718; MS (EI, $\left.70 \mathrm{eV}\right)$ $\mathrm{m} / \mathrm{e} 148\left(\mathrm{M}^{+}, 100\right), 147\left(\mathrm{M}^{+}-\mathrm{H}, 90\right), 104$ (20); HRMS (EI, 70 ev) calcd for $\mathrm{C}_{8} \mathrm{H}_{8} \mathrm{~N}_{2} \mathrm{O}$ 148.0637, obsd 148.0638. Anal. Calcd for $\mathrm{C}_{8} \mathrm{H}_{8} \mathrm{~N}_{2} \mathrm{O}$ : C, 64.85; $\mathrm{H}, 5.54 ; \mathrm{N}$ : 18.91. Found: $\mathrm{C}, 64.68 ; \mathrm{H}$ : 5.42; N: 18.90 .

Typical Synthetic Procedures for S-methyl N-Alkylthiocarbamates. S-Methyl N-Benzylthiocarbamate (36). To a solution of benzylamine $(0.93 \mathrm{~g}, 0.87 \mathrm{mmol})$ and diisopropylamine $(0.89 \mathrm{~g}, 8.8 \mathrm{mmol})$ in THF $(20 \mathrm{~mL})$ at $-78{ }^{\circ} \mathrm{C}$ under nitrogen atmosphere was added n-BuLi (1.6 M, $10.9 \mathrm{~mL}, 17.5$ $\mathrm{mmol}$ ) in hexane. After addition, the solution was stirred at -78 ${ }^{\circ} \mathrm{C}$ for $0.5 \mathrm{~h}$, followed by addition of a solution of DMDTC (1.07 $\mathrm{g}, 8.8 \mathrm{mmol}$ ). The solution was then allowed to react at room temperature for $20 \mathrm{~h}$. The reaction was quenched by pouring it into a mixture of ice-dilute $\mathrm{HCl}$ solution. The crude solid was dissolved into EtOAc, washed with aqueous $\mathrm{Na}_{2} \mathrm{CO}_{3}$ and brine, dried over anhydrous $\mathrm{MgSO}_{4}$, concentrated under reduced pressure, and recrystallized from hexane to provide $\mathbf{3 6}$ as colorless crystals $(0.95 \mathrm{~g}, 62 \%)$ : $\mathrm{mp} 76-79{ }^{\circ} \mathrm{C}$; ${ }^{1} \mathrm{H}$ NMR (300 $\left.\mathrm{MHz}_{2} \mathrm{CDCl}_{3}\right) \delta 7.20-7.34(\mathrm{~m}, 5 \mathrm{H}), 5.67$ (bs, $\left.1 \mathrm{H}\right), 4.45$ (d, J = 6 $\mathrm{Hz}, 2 \mathrm{H}), 2.35(\mathrm{~s}, 3 \mathrm{H}) ;{ }^{13} \mathrm{C} \mathrm{NMR}\left(75 \mathrm{MHz} \mathrm{CDCl}_{3}\right) \delta 167.9,137.7$, $128.45,127.4,127.3,45.1,12.2 ; \mathrm{IR}(\mathrm{KBr}) \mathrm{cm}^{-1} 3305,1639 ; \mathrm{MS}$ (EI, $70 \mathrm{eV}) 181\left(\mathrm{M}^{+}, 30\right), 133$ (30), 91 (100); HRMS (EI, $\left.70 \mathrm{eV}\right)$ calcd for $\mathrm{C}_{9} \mathrm{H}_{11} \mathrm{NOS} 181.0562$, obsd 181.0559. Anal. Calcd for $\mathrm{C}_{9} \mathrm{H}_{11}$ NOS: C, 59.64; $\mathrm{H}, 6.12 ; \mathrm{N}, 7.73$. Found: $\mathrm{C}, 59.65 ; \mathrm{H}, 6.12$; N, 7.78.

S-Methyl N,N'-(p-Xylylene)bis(thiocarbamate) (39). Recrystallization from EtOAc-hexane yielded 39 as colorless crystals: $\mathrm{mp} 201-203^{\circ} \mathrm{C} ;{ }^{1} \mathrm{H}$ NMR $(200 \mathrm{MHz}$, DMSO-d 6 ) $\delta 8.64$ (broad triplet, $\mathrm{J}=6.0 \mathrm{~Hz}, 2 \mathrm{H}), 7.17(\mathrm{~s}, 4 \mathrm{H}), 4.25(\mathrm{~d}, \mathrm{~J}=6.0 \mathrm{~Hz}$, $4 \mathrm{H}), 2.20(\mathrm{~s}, 6 \mathrm{H}) ;{ }^{13} \mathrm{C} \mathrm{NMR}\left(50 \mathrm{MHz}, \mathrm{DMSO}-\mathrm{d}_{6}\right) \delta 166.4,137.8$, 127.3, 43.7, 11.6; IR (KBr) cm ${ }^{-1} 3244,1629 ; \mathrm{MS}$ (EI, $\left.70 \mathrm{eV}\right) 284$ $\left(\mathrm{M}^{+}, 1\right), 236$ (10), 193 (46), 188 (16), 180 (37), 146 (100), 91 (48); HRMS (EI, $70 \mathrm{eV}$ ) calcd for $\mathrm{C}_{12} \mathrm{H}_{16} \mathrm{~N}_{2} \mathrm{O}_{2} \mathrm{~S}_{2} 284.0653$, obsd 284.0652. Anal. Calcd for $\mathrm{C}_{12} \mathrm{H}_{16} \mathrm{~N}_{2} \mathrm{O}_{2} \mathrm{~S}_{2}$ : C, 50.70; $\mathrm{H}, 5.68 ; \mathrm{N}$, 9.86. Found: C, 51.30; $\mathrm{H}, 5.74 ; \mathrm{N}, 9.70$.

Typical Synthetic Procedures for Unsymmetrical Ureas from S-Methyl N-Alkylthiocarbamates. N-Benzyl-N'-tetrahydrofurfurylurea (38). To a stirred solution of S-methyl $\mathrm{N}$-benzylthiocarbamate (36) $(0.11 \mathrm{~g}, 0.63 \mathrm{mmol})$ in methanol $(2$

(13) Hall, H. K. J r.; El-Shekiel, A. J . Org. Chem. 1980, 45, 5325. (14) Yoshida, T.; Kambe, N.; Murai, S.; Sonoda, N. J . Org. Chem. 1987, 52, 1611 .
$\mathrm{mL}$ ) was added tetrahydrofurfurylamine $(0.12 \mathrm{~g}, 1.1 \mathrm{mmol})$. After being heated at $60{ }^{\circ} \mathrm{C}$ for $24 \mathrm{~h}$, the reaction mixture was concentrated under reduced pressure, providing a crude solid. Recrystallization of the solid from $\mathrm{CHCl}_{3}$-hexane gave $\mathbf{3 8}$ as colorless crystals $(0.14 \mathrm{~g}, 88 \%): \mathrm{mp} 78-80{ }^{\circ} \mathrm{C}$; ${ }^{1 \mathrm{H}} \mathrm{NMR}(300$ $\left.\mathrm{MHz}_{2} \mathrm{CDCl}_{3}\right) \delta 7.18-7.30(\mathrm{~m}, 5 \mathrm{H}), 5.86$ (broad triplet, $1 \mathrm{H}$ ), 5.55 (broad triplet, $1 \mathrm{H}), 4.27(\mathrm{~d}, \mathrm{~J}=6 \mathrm{~Hz}, 2 \mathrm{H}), 3.90(\mathrm{~m}, 1 \mathrm{H}), 3.60-$ $3.80(\mathrm{~m}, 2 \mathrm{H}), 3.39(\mathrm{~m}, 1 \mathrm{H}), 3.03(\mathrm{~m}, 1 \mathrm{H}), 1.75-1.95(\mathrm{~m}, 3 \mathrm{H}), 1.51$ $(\mathrm{m}, 1 \mathrm{H}) ;{ }^{13} \mathrm{C}$ NMR $\left(100 \mathrm{MHz} \mathrm{CDCl}_{3}\right) \delta 158.7,139.3,128.5,127.4$, 127.2, 78.8, 68.0, 44.5, 44.4 28.2, 25.9; IR ( $\mathrm{KBr}) \mathrm{cm}^{-1} 3339,1615$; MS (EI, $70 \mathrm{eV}) 234$ (M+, 31), 190 (17), 164 (40), 151 (20), 106 (58), 91 (80), 71 (100); HRMS calcd for $\mathrm{C}_{13} \mathrm{H}_{18} \mathrm{~N}_{2} \mathrm{O}_{2} 234.136$, obsd 234.1369. Anal. Calcd for $\mathrm{C}_{13} \mathrm{H}_{18} \mathrm{~N}_{2} \mathrm{O}_{2}: \mathrm{C}, 66.64 ; \mathrm{H}, 7.74 ; \mathrm{N}$, 11.95. Found: $\mathrm{C}, 66.67 ; \mathrm{H}, 7.75 ; \mathrm{N}, 11.93$.

N,N"-(p-Xylylene)bis[N'-(3-hydroxypropyl)urea] (40). Recrystallization of $\mathbf{4 0}$ from DMSO-EtOAc afforded colorless crystals: $\mathrm{mp} 219-221{ }^{\circ} \mathrm{C} \mathrm{dec}$; ${ }^{1} \mathrm{H}$ NMR $\left(200 \mathrm{MHz}, \mathrm{DMSO}-\mathrm{d}_{6}\right) \delta$ $7.16(\mathrm{~s}, 4 \mathrm{H}), 6.28(\mathrm{t}, \mathrm{J}=6.0 \mathrm{~Hz}, 2 \mathrm{H}), 5.89(\mathrm{t}, \mathrm{J}=6.0 \mathrm{~Hz}, 2 \mathrm{H})$, $4.45(\mathrm{t}, \mathrm{J}=6.0 \mathrm{~Hz}, 2 \mathrm{H}), 4.15(\mathrm{~d}, \mathrm{~J}=6.0 \mathrm{~Hz}, 4 \mathrm{H}), 3.35-3.42(\mathrm{q}$, $\mathrm{J}=6.0 \mathrm{~Hz}, 4 \mathrm{H}), 3.05(\mathrm{q}, \mathrm{J}=6.0 \mathrm{~Hz}, 4 \mathrm{H}), 1.50$ (quintet, $\mathrm{J}=6.0$ $\mathrm{Hz}, 4 \mathrm{H}) ;{ }^{13} \mathrm{C}$ NMR (75 MHz, DMSO-d 6 ) $\delta$ 158.2, 139.2, 126.9, $58.4,42.7,36.4,33.2$; IR 3325, 1606, 1574; FAB (NBA) 339.2 $\left(\mathrm{M}^{+}+\mathrm{H}\right)$. Anal. Calcd for $\mathrm{C}_{14} \mathrm{H}_{22} \mathrm{~N}_{4} \mathrm{O}_{4}$ : C, 56.78; $\mathrm{H}, 7.74 ; \mathrm{N}$, 16.55. Found: $C, 56.55 ; \mathrm{H}, 7.87 ; \mathrm{N}, 16.50$.

N,N"-(p-Xylylene)bis[N'-(2-methylpropyl)urea] (41). Recrystallization from DMSO-EtOAC afforded $\mathbf{4 1}$ as colorless crystals: $\mathrm{mp} 237-240{ }^{\circ} \mathrm{C}$ dec; ${ }^{1} \mathrm{H}$ NMR $(300 \mathrm{MHz}$, DMSO-d 6 ) $\delta$ $7.15(\mathrm{~s}, 4 \mathrm{H}), 6.19$ (broad triplet, $2 \mathrm{H}), 5.92$ (broad triplet, $2 \mathrm{H}$ ), $4.14(\mathrm{~d}, \mathrm{~J}=6 \mathrm{~Hz}, 4 \mathrm{H}), 2.82(\mathrm{t}, \mathrm{J}=6 \mathrm{~Hz}, 4 \mathrm{H}), 1.60(\mathrm{~m}, 2 \mathrm{H}), 0.81$ $(\mathrm{d}, \mathrm{J}=6.6 \mathrm{~Hz}, 12 \mathrm{H}) ;{ }^{13} \mathrm{C} N M R\left(50 \mathrm{MHz}, \mathrm{DMSO}-\mathrm{d}_{6}\right) \delta 158.1$, 139.2, 126.9, 46.8, 42.6, 28.6, 20.0; IR (KBr) cm-1 3318, 1613, 1562; $\mathrm{FAB}$ (NBA) $335.2\left(\mathrm{M}^{+}+\mathrm{H}\right)$. Anal. Calcd for $\mathrm{C}_{18} \mathrm{H}_{30} \mathrm{~N}_{4} \mathrm{O}_{2}$ : C, 64.64; $\mathrm{H}, 9.04 ; \mathrm{N}, 16.75$. Found: $\mathrm{C}, 64.36 ; \mathrm{H}$, 8.97; N, 16.70.

N,N"-(p-Xylylene)bis(N'-benzylurea) (42). Recrystallization from DMSO-EtOAc afforded $\mathbf{4 2}$ as colorless crystals: $\mathrm{mp}$ 258-261 ${ }^{\circ} \mathrm{C} \mathrm{dec} ;{ }^{1} \mathrm{H}$ NMR (300 MHz, DMSO- $\left.\mathrm{d}_{6}\right) \delta 7.20-7.33(\mathrm{~m}$, $10 \mathrm{H}), 7.17(\mathrm{~s}, 4 \mathrm{H}), 6.39(\mathrm{~m}, 4 \mathrm{H}), 4.21(\mathrm{~d}, \mathrm{~J}=6 \mathrm{~Hz}, 4 \mathrm{H}), 4.19(\mathrm{~d}$, $J=6 \mathrm{~Hz}, 4 \mathrm{H}) ;{ }^{13} \mathrm{C} \mathrm{NMR}\left(50 \mathrm{MHz}, \mathrm{DMSO}-\mathrm{d}_{6}\right) \delta 158.0,140.9$, 139.2, 128.2, 128.1, 127.0, 126.6, 42.9, 42.7; IR ( $\mathrm{KBr}) \mathrm{cm}^{-1} 3319$, 1619; $F A B$ (NBA) $403.2\left(M^{+}+H\right)$. Anal. Calcd for $\mathrm{C}_{24} \mathrm{H}_{26} \mathrm{~N}_{4} \mathrm{O}_{2}$ : C, 71.62; $\mathrm{H}, 6.51 ; \mathrm{N}, 13.91$. Found: $\mathrm{C}, 71.52 ; \mathrm{H}$, 6.50; N, 13.90 .

Acknowledgment. We thank the National Science Council of the Republic of China for the financial support (NSC 85-2113-M-002-013 and NSC 85-2815C002-01-010M).

Supporting Information Available: ${ }^{1 \mathrm{H}}$ and ${ }^{13} \mathrm{C}$ NMR spectra of DMDTC and compounds 4, 6, 8, 12, 14, 16, 19, 22, $\mathbf{2 4}, \mathbf{2 9}, \mathbf{3 1}, \mathbf{3 3}, \mathbf{3 5}, \mathbf{3 6}$, and $\mathbf{3 8}-\mathbf{4 2}$ (38 pages). This material is contained in libraries on microfiche, immediately follows this article in the microfilm version of the journal, and can be ordered from the ACS; see any current masthead page for ordering information.

J 09522825 